\title{
Neuropsicologia do desenvolvimento da memória: da pré-escola ao período escolar
}

\author{
Neuropsicología del desarrollo de la memoria: de la edad preescolar al período escolar \\ Neuropsychologie de la mémoire chez l'enfant: de l'âge préscolaire à l'âge scolaire \\ Neuropsychology of memory development: from preschool to school age
}

\section{Luciana Brooking Teresa Dias ${ }^{1} \&$ J. Landeira-Fernandez ${ }^{\mathbf{1} 2}$}

\author{
${ }^{1}$ Pontifícia Universidade Católica do Rio de Janeiro, Brasil. ${ }^{2}$ Universidade Estácio de Sá, Brasil.
}

Agradecimentos: Os autores agradecem aos dois pareceristas ad hoc pelas sugestões de modificações feitas ao texto original. Agradecem também a CAPES (LBTD foi bolsista de mestrado da CAPES de março de 2009 a fevereiro de 2011) e ao CNPq (JLF é pesquisador bolsista produtividade do CNPq).

\begin{abstract}
Resumo
A memória é uma função cognitiva complexa formada por diferentes componentes que se relacionam entre si. Em face dos avanços e das crescentes demandas nas áreas de neuropsicologia do desenvolvimento e escolar, especialmente na América Latina, torna-se importante promover estudos de revisão teórica em busca de um entendimento mais aprofundado sobre como se desenvolvem os diferentes tipos, componentes e sistemas mnemônicos de crianças na fase pré- e escolar assim como em períodos de desenvolvimento subsequentes. Utilizando-se como critério o tempo em que uma informação fica armazenada com o indivíduo, pode-se classificar a memória em sensorial, de curto e de longo prazo. A memória de trabalho representa um tipo de memória de curto prazo, em relação constante com a de longo prazo, formada por alça fonológica, esboço visuoespacial, executivo central e retentor episódico. A memória de longo prazo apresenta um componente explícito e implícito. A memória se desenvolve ao longo da vida do indivíduo a partir das relações entre aspectos biológicos e sociais. Bebês já apresentam algum tipo de memória, ainda que rudimentar, reproduzindo ações após intervalos de tempo, como se fosse o início da memória explícita; reconhecem faces e eventos familiares; e apresentam memória implícita, que não sofre muita alteração ao longo do desenvolvimento. Na pré-escola, quando a criança começa a apresentar maturação do sistema nervoso, a verbalizar e a desenvolver um pensamento mais abstrato, juntamente com a ajuda dos pais na estimulação de sua linguagem, a memória explícita assim como a memória de trabalho começam a se tornar mais sofisticadas, permitindo que informações possam ser mais bem organizadas. Na fase escolar, a memória já se encontra mais desenvolvida, principalmente em crianças mais velhas. Essa revisão teórica pode contribuir para a organização do importante conhecimento sobre as bases mnemônicas para uma melhor compreensão das dificuldades que podem ocorrer na infância, assim como para a promoção de estratégias de estimulação de componentes mnenônicos nesta fase.

Palavras-chave: Memória; cognição; desenvolvimento; ontogênese; pré-escola; escola.
\end{abstract}

\section{Resumen}

La memoria es una función cognitiva compleja formada por diferentes componentes que se relacionan entre sí. Dados los avances y las demandas crecientes, especialmente en América Latina, en las áreas de la neuropsicología del desarrollo y del ámbito escolar, resulta importante realizar estudios de revisión teórica en busca de un mayor entendimiento del desarrollo de los diferentes tipos, componentes y sistemas de memoria en niños en fase preescolar, así como también en los períodos posteriores del desarrollo. Si se usa como criterio el tiempo que una información se almacena, la memoria se puede clasificar en sensorial, de corto y de largo plazo. La memoria de trabajo constituye un tipo de memoria de corto plazo, la cual está en relación constante con la de largo plazo. Está formada por el bucle fonológico, la agenda viso-espacial, el ejecutivo central y el retén episódico. La memoria de largo plazo tiene un componente explícito y uno implícito. La memoria se desarrolla a lo largo de la vida de un individuo a partir de las relaciones entre aspectos biológicos y sociales.

Artigo recibido: 13/08/2010; Artigo revisado: 13/09/2010; Artigo aceito: 08/04/2011.

J. Landeira-Fernandez, Departamento de Psicologia, Pontifícia Universidade Católica do Rio de Janeiro. Luciana Brooking Teresa Dias, Curso de Psicologia, Universidade Estácio de Sá.

J. Landeira-Fernandez, Núcleo de Neuropsicologia Clínica e Experimental, Laboratório de Análise de Dados, Departamento de Psicologia, Pontifícia Universidade Católica do Rio de Janeiro, Rio de Janeiro, Brasil, CEP 22453-900.

E-mail: landeira@puc-rio.br

DOI: $10.5579 / \mathrm{rnl} .2011 .0061$ 
Los bebés ya presentan algún tipo rudimentario de memoria al reproducir acciones luego de un cierto intervalo de tiempo, como si fuera el inicio de la memoria explícita. También reconocen rostros y eventos familiares y poseen memoria implícita, yaque no sufre muchos cambios a lo largo del desarrollo. En preescolar, con la maduración del sistema nervioso, los niños verbalizan y desarrollan un pensamiento más abstracto. Con la ayuda de sus padres para la estimulación de su lenguaje, la memoria explícita y la de trabajo comienzan a complejizarse, permitiendo una mejor organización de la información. En la etapa escolar la memoria se encuentra más desarrollada, principalmente en niños mayores. Esta revisión teórica contribuye a organizar vasto conocimiento sobre las bases de la memoria a fin de comprender mejor las dificultades que pueden ocurrir en la infancia. Además, para la promoción de estrategias de estimulación de los componentes de la memoria en esta etapa del desarrollo.

Palabras-clave: Memoria; cognición; desarrollo; ontogénesis; preescolar; escuela.

\section{Résumé}

La mémoire est une fonction cognitive constituée de différentes composantes qui interagissent entre elles. À la lumière des avancées et des demandes accrues dans le domaine de la neuropsychologie développementale et scolaire, particulièrement en Amérique latine, il est important d'établir une revue des études théoriques et expérimentales afin de mieux comprendre comment se développent les différents systèmes mnésiques durant la période préscolaire ainsi que celle qui lui succède. Durant le maintien des informations en mémoire, la trace mnésique peut être classifiée en tant que mémoire sensorielle, mémoire à court terme ou mémoire à long terme. La mémoire de travail est considérée comme un type de mémoire à court terme, en relation constante avec la mémoire à long terme. Elle est formée de la boucle phonologique, du calepin visuospatial, de l'administrateur central et du "buffer épisodique". La mémoire à long terme possède une composante explicite et implicite. La mémoire se développe tout au long de la vie de l'individu, à partir des relations entre des aspects biologique et sociaux. La mémoire est déjà présente chez les nouveaux-nés, bien que rudimentaire. Elle consiste en la reproduction d'actions après un certain délai ou en la reconnaissance de visages et d'événements familiaux, comme s'il s'agissait des prémisses de la mémoire explicite, mais c'est surtout la mémoire implicite qui serait déjà présente chez les nouveaux-nés car ce type de mémoire évolue très peu au cours du développement. A l'âge préscolaire, lorsque les enfants commencent à montrer un maturation du système nerveux suffisante pour verbaliser et développer une pensée plus abstraite, grâce à l'aide de la stimulation du langage par les parents, la mémoire explicite et la mémoire de travail deviennent plus sophistiquées, ce qui permet une meilleure organisation de l'information. À l'école, la mémoire est donc déjà plus développée, surtout chez les enfants plus âgés. Cette révision théorique peut contribuer à l'organisation des connaissances qui devraient être considérées pour une meilleure compréhension des difficultés qui peuvent apparaître durant l'enfance, ainsi que pour des stratégies promouvant la stimulation des composantes mnésiques dans cette phase.

Mots-clès : Développement de la mémoire ; ontogenèse ; préscolaire ; cognition.

\section{Abstract}

Memory is a complex cognitive function made up of different components that interact. In light of the advances and the increasing demands in the areas of developmental and school neuropsychology, especially in Latin America, it is important to review theoretical and experimental studies, so that we can better understand how different memory systems develop during pre-school as well as in subsequent periods of development. From the time that information is stored, memory can be classified into sensory memory, short and long term. Working memory is a type of short-term memory, in constant relation with the long term, formed by the phonological loop, sketchpad visuospatial, the central executive and episodic buffer. The long-term memory has an explicit and implicit component. The memory develops over the life of the individual from the relations between social and biological aspects. Babies already have some kind of memory, though rudimentary, reproducing actions after time intervals, as if it were the beginning of explicit memory, recognize faces and family events, and show implicit memory, which does not undergo much change during development. In preschool, when the child begins to show maturation of the nervous system to verbalize and develop a more abstract thought, with the help of parents in stimulating their language, explicit memory and working memory are becoming more sophisticated, allowing information to be better organized. At school, the memory is already more developed, primarily in older children. This theoretical approach can contribute to the organization of important knowledge bases mnemonic that should be included for a better understanding of the difficulties that may occur in childhood, as well as strategies to promote stimulation of memory components in this phase.

Keywords: Memory; cognition; development; ontogeny; preschool; school.

A memória desenvolve-se graças à íntima relação entre aspectos biológicos e sociais. Tal desenvolvimento se inicia na fase pré-natal. Após o nascimento, a memória representa uma das principais funções mentais e determina a forma como o indivíduo irá se desenvolver ao longo da sua vida. É através dessa função que o passado se torna presente, permitindo assim que ações sejam adaptativas e que os indivíduos possam lidar de forma adequada com as demandas do cotidiano.

Esta função cognitiva acompanha as diversas mudanças que ocorrem com as crianças, principalmente durante o período pré-escolar assim como o momento em que a criança ingressa na escola. É lá que ela adquire novas experiências possibilitando modificações associadas à extensão do vocabulário, ampliação de conceitos e elaboração de melhores estratégias para resolver problemas, juntamente com a maturação do sistema nervoso e a aprendizagem formal fornecida pelas escolas (Mello \& Xavier, 2006).

Em face dos avanços e das crescentes demandas nas áreas de neuropsicologia do desenvolvimento e escolar, especialmente na América Latina, torna-se importante promover estudos de revisão teórica em busca de um entendimento mais aprofundado sobre como se desenvolvem os diferentes tipos, componentes e sistemas mnemônicos de crianças na fase pré- e escolar, assim como em períodos de desenvolvimento subsequentes. Demandas crescentes da neuropsicologia do desenvolvimento têm impacto direto no processo escolar, uma vez que falhas nesse sistema podem provocar prejuízos no processo de aprendizagem, na leitura e compreensão de um texto, e na resolução de problemas de matemática, assim como na aquisição e na produção de vocabulário. Portanto, um comprometimento de sistemas mnemônicos relaciona-se de forma clara com as dificuldades de aprendizagem e ao baixo rendimento escolar. O estudo do 
desenvolvimento dessas funções pode auxiliar no processo de diagnóstico e eventualmente de forma mais clara em intervenções dentro do ambiente escolar.

\section{Memórias: conceitos, tipos, componentes e correlatos neurais}

Nesse contexto de desenvolvimento da memória, cabem algumas distinções. A memória é uma função cognitiva complexa composta por sistemas distintos, mas que se relacionam entre si (Luria, 1981). Atualmente já é consenso que os diversos tipos de memória estão associados a diferentes áreas cerebrais que funcionam interligadas formando circuitos neurais específicos (Gazzaniga \& Heatherton, 2005; Kandel, 2009).

Classicamente, a memória pode ser dividida em estágios, que se classificam conforme o tempo de retenção ou armazenamento de uma informação: muito rápido (na ordem de milissegundos, denominada de memória sensorial), de curto e de longa duração. A memória de curto prazo é aquela que apresenta armazenamento temporário de poucas informações por curto intervalo de tempo advindas da memória sensorial ou da memória de longo prazo. Cabe ressaltar que dentro do contexto de curto prazo há a memória de trabalho ou operacional. Essa função mantém uma informação na consciência, manuseando-a com objetivo de resolver algum tipo de problema.

A memória de trabalho subdivide-se em quatro subsistemas: alça fonológica (armazenamento temporário e manipulação das informações verbais, sejam auditivas, de leitura, ou de repetição de estímulos linguísticos); esboço visuoespacial (armazenamento e manipulação visual e espacial, como detalhes de um objeto); retentor episódico (subsistema estudado mais recentemente, recupera a informação de forma consciente e trabalha as atividades cognitivas mais complexas); executivo central (sistema atencional que atua na seleção e manipulação da informação nos outros subsistemas, para também ser enviada à memória de longo prazo, agindo como um controlador e buscando informações já armazenadas na memória de longo prazo) (Baddeley, 2000, 2002, 2003, 2007; 2009).

Finalmente, a memória de longo prazo tem a capacidade de armazenar informações por períodos de tempo bem mais longos, na ordem de minutos, horas, dias, semanas, meses, anos. Esse tipo de memória também apresenta diversos componentes. A memória explícita ou declarativa depende de processos conscientes e é passível de verbalização. Ela se subdivide em episódica e semântica. A memória episódica tem relação com as experiências vividas e que se pode delimitar no tempo e espaço. Está mais envolvida com o hipocampo, que tem a função de facilitar o processo de aquisição de informações (Landeira-Fernandez, 2006; Lombroso, 2004; Squire \& Zola-Morgan, 1991; Squire et al., 1992; Tulving, 2002). Já a memória semântica tem relação com conhecimentos gerais, que nem sempre têm ligação com os fatos vividos, como conceitos e teorias. Ela está mediada por estruturas neocorticais anteriores e posteriores (Moscovitch et al., 2005).

A memória episódica e a memória semântica representam componentes independentes da memória de longo prazo. Por via de regra, a primeira possibilita a formação da segunda, fornecendo conteúdo para isso, enquanto a semântica facilita que novas memórias episódicas sejam formadas (Greenberg \& Verfaellie, 2010). Embora estas duas formas de memória sejam relativamente independentes, elas interagem a todo momento durante a execução das diversas funções cognitivas. Por exemplo, uma informação episódica pode ser mais facilmente evocada caso ela tenha sido processada de forma semântica (Greve, van Rossum \& Donaldson, 2007).

Alguns autores diferenciam a memória autobiográfica da episódica, pois aquela só envolve fatos autobiográficos. Fivush (2011) distingue a característica do "onde", "o quê" e "quando" presentes na memória episódica da consciência de quando o fato acontece para si, suas características, seu próprio tempo, considerando a relação com sua cultura, linguagem e a sociedade em que se está inserido. Dessa forma, situa-o em relação a si mesmo, ao seu grupo de relações, à sua saúde física e psicológica e ao seu contexto (Conway \& Pleydell-Pearce, 2000; Fivush, 2011; Nelson \& Fivush, 2004). Ela contém componentes de memória semântica (Moscovitch et al., 2005) e da episódica, que também constituem a memória autobiográfica; juntas formarão a memória de longo prazo (Conway, 2009; Morrison \& Conway, 2010).

A memória implícita caracteriza-se pelo fato de não depender de processos conscientes e de difícil verbalização. Envolve procedimentos, habilidades motoras, e hábitos. Acredita-se que a memória implícita recruta estruturas cuja ativação está relacionada ao processamento motor, tais como os núcleos da base, o estriato, o cerebelo, o córtex motor, e o córtex pré-motor.

Desenvolvimento da memória: da primeira à segunda infância, da fase pré-escolar à escolar

Em uma revisão da literatura, Carneiro (2007) apresenta uma série de evidências clínicas e experimentais que indicam que a memória sofre inúmeras modificações ao longo da vida, especialmente durante a idade pré-escolar. Em outra revisão, Nelson (1995) apresenta um modelo de desenvolvimento da memória. De acordo com esse modelo, memórias que são processadas na primeira infância estariam mais associadas ao lobo medial temporal, mais precisamente ao hipocampo (tarefas de comando e com pequeno intervalo), ao passo que memórias relacionadas com a idade adulta dependeriam mais de áreas corticais.

Nesta perspectiva ontológica, sabe-se que desde o início da vida já há indícios da presença da memória. Os bebês recordam fatos do passado, porém de forma diferente das crianças e dos adultos (Bauer, 2006). Nessa fase existem duas maneiras de avaliar a memória através de imitação: uma de forma imediata, chamada imitação eliciada e outra, após um intervalo, chamada imitação diferida (Bauer, Wenner, \& Kroupina, 2002; Meltzoff, 1988). A imitação diferida é uma medida de memória não verbal (Barnat, Klein, \& Meltzoff, 1996; Barr, Dowden, \& Hayne, 1996). Ocorre quando o bebê observa várias ações com estímulos-alvo e depois de um intervalo é capaz de reproduzi-la, após 24 horas, marcadas quando se passa da linha de base (Barr et al., 1996; Nelson \& Fivush, 2004; Rovee-Collier \& Cuevas, 2009). Essa é uma medida que vai além do reconhecimento, pois verifica a capacidade de generalizar uma ação, representando um estímulo que foi armazenado (Barnat et al., 1996).

Há evidências de mudanças ao longo do desenvolvimento da memória através da imitação diferida de bebês de 6 a 24 meses, nas quais aqueles com 18 e 24 meses demonstram melhor desempenho, maior do que os de 6 meses; os de 12 ficam em posição intermediária. Porém, se 
houver maior frequência de imitação, os bebês de 6 meses conseguem apresentar bom desempenho de memória diferida (Barr et al., 1996).

Essa habilidade é entendida como o início da memória explícita. Aos 3 meses o bebê já é capaz de apresentar essa habilidade (Rovee-Collier \& Cuevas, 2009). Todavia, ainda não se sabe precisamente quando os bebês começam a demonstrar sinais de desenvolvimento da memória explícita, pois os eventos neurais que fundamentam tal desenvolvimento ainda não estão totalmente esclarecidos. Há possibilidade de que a memória de longo prazo possa se desenvolver no final do primeiro ano de vida (Carver, Bauer, \& Nelson, 2000).

No caso da imitação eliciada, os bebês são encorajados a produzir as sequências das ações-alvo logo após a demonstração, assim como após intervalo curto (Courage \& Howe, 2002), ou seja, é uma imitação imediata. Nesse caso há dados que demonstram semelhanças entre os bebês de 6 a 24 meses (Barr et al., 1996). Bebês com 2 meses de idade e treinados e re-treinados de tempos em tempos, apresentaram claras evidências de sistemas de memória estáveis (RoveeCollier, Hartshorn, \& DiRubbo, 1999). Ainda aos 6 meses o bebê tem sua memória dependente do contexto, ou seja, só reproduzirá um treinamento um tempo depois se estiver no mesmo ambiente treinado. Aos 8 ou 9 meses é que ele se torna independente do contexto, podendo responder em ambiente diferente do treinado. Se mesmo assim não responder, é porque apresenta a memória como na época da codificação, mas se responder de uma maneira no contexto treinado e de outra no testado é porque sua memória se apresenta conforme a idade do teste (Hartshorn \& RoveeCollier, 2003). Como o bebê aos 6 meses esquece-se do treinamento após duas semanas, é necessário ser retreinado até o teste, quando ocorre após intervalo, pelo menos uma vez por mês (Hartshorn \& Rovee-Collier, 2003).

Técnicas de potencial evocado relacionado a eventos assim como tarefas de reconhecimento a objetos familiares e não familiares vem sendo empregadas para o estudo de processos de desenvolvimento da memória em bebê ao longo do primeiro ano de vida (Carver et al., 2000; Nelson \& Collins, 1991). Por exemplo, em um experimento realizado com bebês de 9 meses observou-se alterações em potencial evocado quando conseguiam identificar eventos familiares (Carver et al., 2000). Também aos 9 meses foi detectado que os bebês que discriminavam os diferentes objetos também demonstravam evidência de discriminação no potencial evocado relacionado a eventos, ao contrário dos que não conseguiam (Nelson, 1998).

Com o intuito de investigar os efeitos da imitação em bebês de 9 meses e meio no reconhecimento imediato e tardio, além da evocação na memória de longo prazo, foi registrado potencial evocado relacionado a eventos durante uma tarefa de imitação eliciada. Nesse estudo, observou-se que houve codificação, a imitação melhorou o reconhecimento e a evocação tardia, facilitando o desempenho mnemônico (formas mais fáceis para memorizar e lembrar-se de itens complexos), e que talvez tenha havido participação de neurônios espelho (Lukowski et al., 2005). Nessa idade, os bebês são capazes de reproduzir os estímulos vivenciados imediatamente com novos brinquedos e também após 24 horas de intervalo (Meltzoff, 1988).

Diversas evidências indicam também que o bebê, ao longo do seu primeiro ano de vida, já consegue se lembrar de fatos passados após um intervalo relativamente longo de tempo. Aos 13 ou 16 meses não se encontraram evidências de haver memória verbal relativamente estável em oposição a bebês (Bauer et al., 2002). Esses dados sugerem que existe um formato de organização não-verbal antes da verbalização.

Em outro estudo, crianças de 3 anos não foram capazes de evocar informações apresentadas aos 6 meses de idade (época do primeiro encontro), mas demonstraram sucesso na evocação de outras informações adquiridas aos 20 meses de idade. Tanto os bebês de 16 meses quanto os de 20 meses evocaram após intervalo de 6 e 12 meses. Dessa forma, pode-se constatar que mesmo as crianças que ainda não falam no período da aquisição da informação são capazes de evocar essas informações mais tarde após a aquisição da linguagem (Bauer, Kroupina, Schwade, Dropik, \& Wewerka, 1998).

Os dados obtidos a partir de estudos com crianças indicam que o desenvolvimento da linguagem no período da pré-escola é fundamental para processos mais elaborados da memória (Carneiro, 2007; Nelson \& Fivush, 2004). Nesse contexto, o desenvolvimento da habilidade narrativa entre $2 \mathrm{e}$ 5 anos é essencial para a memória autobiográfica (Fivush \& Schwarzmueller, 1998). Nos primeiros anos da pré-escola, os processos de memória explícita estão envolvidos com processos relacionados com o planejamento mental de tarefas permitindo assim uma melhora na capacidade de evocação de informações (Greenbaum \& Graf, 1989). Na verdade, existem evidências indicando que crianças antes dos 2 anos de idade não apresentam grupamentos de representações mentais, uma habilidade importante para a memória explícita, uma vez que possibilita grupamentos nos quais as informações estão associadas, facilitando sua evocação (Greenbaum \& Graf, 1989).

A memória autobiográfica se desenvolve de acordo com os estímulos recebidos que rodeiam a criança, principalmente da mãe, da sua sociedade, da sua cultura. Conforme ela vai organizando sua narrativa e vai sendo estimulada com ajuda externa, sua própria memória vai se estruturando. Apenas no final da adolescência e no início da fase adulta é que ela se consolida, apesar de iniciar seu desenvolvimento na pré-escola (Fivush, 2011).

A linguagem tem também um papel crítico no desenvolvimento da memória autobiográfica, uma vez que essa função cognitiva ajuda na organização de informações, permitindo a troca de experiências entre as crianças, colaborando na estruturação das suas próprias vivências, e ajuda na consciência da representação do que viveu (Nelson \& Fivush, 2004). Juntamente com a linguagem, a atenção precisa estar mais desenvolvida para possibilitar o melhor funcionamento da memória (Courage \& Howe, 2002).

A capacidade para armazenar informações na memória de longo prazo está diretamente associada à idade da criança. Crianças mais velhas apresentam melhor codificação, o que também facilita sua evocação das informações armazenadas. Essas características estão relacionadas ao desenvolvimento de estruturas neurais, especialmente do córtex pré-frontal e do giro denteado do hipocampo, estruturas que sofrem influência da cultura em que a criança está inserida (Bauer, 2008).

De fato, sabe-se que o processo de maturação apropriado dessas estruturas neurais é fundamental para a expressão adaptada dessas funções mnemônicas. Dessa forma, processos de plasticidade cerebral, responsáveis pelo fortalecimento de circuitos neurais, permitem com que 
diversos componentes e sistemas mnemônicos possam atingir melhor desempenho conforme a criança vai se desenvolvendo (Brehmer, Li, Müller, Von Oertzen, \& Lindenberger, 2007). Essa melhora se dá tanto em relação aos componentes visuais quanto aos verbais (Duncan, Whitney, \& Kunen, 1982). Esse fato pode ser devido ao tipo de processamento sensorial, no qual a percepção da criança se diferencia da do adulto, e não graças a diferenças em funções relacionadas com fatores cognitivos (Wilburn \& Feeney, 2008). De fato, crianças com lobo frontal imaturo têm dificuldade de relatar as circunstâncias em que aprendeu uma nova informação (Gazzaniga \& Heatherton, 2005). Portanto, processos de plasticidade neural devem ser estimulados através de atividades cognitivas que possam exercitar esses sistemas mnemônicos, especialmente durante a primeira infância, que representa o período crítico de desenvolvimento da criança. Período crítico porque a experiência durante os primeiros anos de vida tem um efeito estruturante no desenvolvimento infantil, em oposição a efeitos circunstanciais da experiência fora deste período. De fato, o período crítico representa um momento em que o sistema nervoso está extremamente sensível aos processos de aprendizagem produtores de alterações permanentes e definitivas em determinadas estruturas neurais.

Em relação à memória declarativa, crianças de 12 anos têm desempenho melhor que as de 8 anos, evidenciando a diversidade de sistema de memória, no qual a memória declarativa melhora com a idade, juntamente com a maturação do hipocampo, diencéfalo e córtex temporal (DiGiulio, Seinderberg, O’Leary, \& Raz, 1994), além do sistema límbico (McKee \& Squire, 1993). De fato, aspectos emocionais representam aspectos importantes na formação de novas memórias. Por exemplo, crianças que passaram pelos mesmos eventos aos 3 anos, quando tinham 7, 8 e 9 anos tinham que recordar os eventos vividos, sendo que alguns desses eventos haviam sido reforçados. Os resultados mostraram que os eventos reforçados foram recordados com certa facilidade ao passo que aqueles que não sofreram qualquer reforço simplesmente foram esquecidos (Abbema \& Bauer, 2005).

Outro aspecto muito importante no desenvolvimento da memória é a comunicação entre mães e seus filhos. Essa interação, por meio da fala, possibilita uma organização do relato da criança, o entendimento da passagem do tempo e compreensão dos outros; além disso, o senso de quem são, que também é passado na relação com os outros, também sofre influência da cultura em que estão inseridos. Essa formação está presente na memória autobiográfica do sujeito, transmitindo sua história ao meio em que vive (Burch, Austin, \& Bauer, 2004; Fivush \& Nelson, 2004; Fivush \& Schwarzmueller, 1998).

Há dois estilos de conversa das mães com seus filhos: as conversas mais elaboradas, que falam sobre o passado, e as menos elaboradas, que falam menos sobre o passado e não têm tanta riqueza de detalhes (Han, Leichtman, \& Wang, 1998; Larkina, Güler, Kleinknecht, \& Bauer, 2008); há ainda aquelas que falam sobre o futuro (Nelson \& Fivush, 2004). Dessa forma, vão se formando os conceitos temporais da criança, assim como aqueles sobre si e sobre os outros (Nelson \& Fivush, 2004). A relação entre memória e linguagem mostra-se, então, inerente ao processamento dos componentes de ambas as funções cognitivas.
No caso das conversas mais elaboradas, mais facilidade a criança terá em falar de forma mais consistente sobre o passado, e essa característica apresentará grande variação na fala das crianças, inclusive culturais. Isso engloba crenças dos pais, regras e valores sociais (Han et al., 1998; Larkina et al., 2008).

A criança cuja mãe organiza seu discurso em categorias é mais propensa a ter melhor codificação e evocação de informações (Larkina et al., 2008). Observa-se que, em geral, meninas apresentam maior elaboração no diálogo com o pai aos 3, 6, 5 e 10 anos. Elas usam mais detalhes, passando um relato com mais vivência, talvez pela maneira diferenciada do diálogo dos pais, ao invés das mães, com elas, elaborando melhor com as filhas, apesar da presença também de melhor elaboração dos filhos com os pais do que com as mães (Reese, Haden, \& Fivush, 1996). Estas peculiaridades do desenvolvimento comunicativo e mnemônico verbal quanto ao gênero podem justificar as evidências de desenvolvimento mais acelerado da inteligência verbal em meninas do que em meninos até a adolescência (Kramer, Delis, Kaplan, O'Donnell, \& Prifitera, 1997).

No entanto, há estudos que não corroboram uma visão consensual sobre tais diferenças. Aos 8 anos, as crianças que passaram por conversas específicas com suas mães sobre fatos vividos aos 40, 46, 58 e 70 meses - e aos 40 e 70 também com pais - lembraram mais dos eventos aos 70 meses e com mais riqueza verbal. Apesar de as meninas evocarem mais detalhes, não houve diferença estatística significativa (Fivush \& Schwarzmueller, 1998).

Em outro estudo perguntou-se para adultos sobre memórias de eventos vividos na infância. Os resultados indicaram que as maiores lembranças de 2 anos de idade foram os nascimentos e hospitalizações; os de 3 anos foram de mudanças e mortes na família; não houve nenhuma lembrança de 1 ano. Um fato curioso foi que aqueles que tiveram muito contato com experiências vividas por meio de fotos ou relatos da família tenderam a ter menos lembranças de eventos ocorridos mais cedo em suas vidas (Usher \& Neisser, 1994).

Esses dados sugerem que crianças, desde cedo, são capazes de codificar, armazenar e evocar informações. E quanto mais velhas, melhor se recordam dos fatos (Abbema \& Bauer, 2005). Essa memória, porém, ainda é rudimentar nos bebês (Gazzaniga \& Heatherton, 2005). Crianças muito novas ainda não têm a memória declarativa formada. Ela se desenvolve aos poucos, a partir do primeiro ano (Nelson \& Fivush, 2004), e só com a linguagem é que podem relatar os fatos conscientes (Bauer et al., 1998).

No caso da memória de trabalho, sabe-se que sua organização em crianças entre 4 a 11 anos pode se dar de forma consistente e estável, sugerindo que as funções cognitivas já estão estabelecidas desde cedo (Alloway, Gathercole, \& Pickering, 2006). Porém, há mais estudos sobre seus subsistemas, o que prejudica a obtenção de informações sobre seu desenvolvimento como um todo e suas mudanças ao longo das idades (Gathercole, Pickering, Ambridge, \& Wearing, 2004). Em um estudo sobre desenvolvimento desse tipo de memória ao longo das idades, verificou-se que os subsistemas vão se aprimorando a partir dos 4 anos até a adolescência, e que aos 6 anos eles já estão organizados da maneira que funcionarão na fase adulta. $\mathrm{O}$ esboço visuoespacial está mais ligado ao executivo central do que à 
alça fonológica, apesar de se apresentarem independentes (Gathercole et al., 2004).

Em avaliação de memória de trabalho de crianças de 7 a 17 anos com problemas aritméticos, os mais velhos tiveram melhor desempenho e aqueles que resolviam mais problemas dentro do tempo estabelecido apresentavam melhor resultado na evocação. $\mathrm{O}$ estudo também mostrou que quanto maior a quantidade de erros, maior era o esquecimento (Towse, Hitch, \& Hutton, 2002).

Crianças com uma maior amplitude de armazenamento da alça fonológica têm melhor capacidade de armazenar informações de palavras na memória de longo prazo (Gathercole, Hitch, Service, \& Martin, 1997). A amplitude de armazenamento de crianças é menor que dos adultos, mas não se sabe como isso afeta a aquisição e retenção das informações (Nelson, 2007). A amplitude de memória foi maior nas idades mais avançadas em estudo com crianças de 5 a 12 anos (Korkman, Kemp, \& Kirk, 2001).

Prejuízos na memória de trabalho podem levar a dificuldades de aprendizagem, fato que não depende da inteligência, ou seja, a inteligência não é um fator crucial no bom desempenho da memória de trabalho (Maehler \& Schuchardt, 2009; Uehara e Landeira-Fernandez, 2010). Por exemplo, problemas de leitura assim como na compreensão de leitura também parecem estar relacionados a limitações no funcionamento da alça fonológica. De fato, existem evidências mostrando que à medida que a criança desenvolve a capacidade de processamento fonológico, os problemas de leitura começam a desaparecer.

Deficiências na aprendizagem de matemática também parecem ser uma consequência de um prejuízo no funcionamento da memória de trabalho em crianças. Diferentes componentes da memória de trabalho desempenham papel fundamental para a resolução de problemas aritméticos. Por exemplo, crianças com problemas de aprendizagem em matemática apresentam déficits no funcionamento da alça fonológica e no esboço visuoespacial. Esses dados sugerem que a representação visuoespacial de informação numérica e os aspectos fonológicos necessários para decompor e compreender o problema matemático podem estar relacionados à origem desse problema de aprendizagem.

Problemas no executivo central também podem estar associados a déficits na aprendizagem de matemática. A dificuldade para coordenar ações mentais necessárias para a solução de um problema matemático certamente é um fator limitante para a resolução desse tipo de tarefa cognitiva. Por exemplo, foi observado que indivíduos com baixo desempenho em tarefas que envolvem cálculos matemáticos, devido à ansiedade elevada induzida por essas tarefas, apresentam também uma menor capacidade no executivo central (Ashcraft \& Kirk, 2001; De Smedt et al., 2009).

Finalmente, existem várias evidências que mostram uma importante relação entre o sono e a memória de trabalho bem como outras funções cognitivas. Por exemplo, problemas de respiração durante o sono, como o ronco, geram problemas neurocognitivos nas crianças (Blunden, Lushington, Kennedy, Martin, \& Dawson, 2000). Um estudo realizado com 81 crianças mostrou que há uma relação entre o distúrbio obstrutivo do sono e prejuízos atenção, aprendizagem e memória (Uema, Pignatari, Fujita, \& Moreira, 2007). Outro estudo com objetivo de avaliar o desempenho neurocognitivo e comportamental de crianças de 5 a 10 anos com queixa de ronco demonstrou que crianças com ronco foram piores em atenção seletiva e sustentada; secundariamente, apresentaram prejuízo na memória, evidenciando que o desempenho neurocognitivo das crianças com ronco foi mais reduzido do que o controle (Blunden et al., 2000). Dessa forma, orientar os pais para que observem o sono dos filhos pode oferecer informações preciosas em situações clínicas em que a criança apresenta problemas em um ou vários sistemas cognitivos.

\section{Considerações finais}

A partir desta revisão teórica, consolida-se a noção cada vez mais forte na literatura de que a memória não é um processo unitário. Além de representar uma função extremante complexa, ela apresenta diversos componentes. Portanto deve-se distinguir os diversos tipos, subprocessos e etapas da memória, em busca de um melhor entendimento de como ela se desenvolve na infância. De fato, cada um desses componentes desenvolve-se de forma singular e específica.

A memória avança juntamente com o desenvolvimento biológico em compasso com as relações sociais que a criança vai formando em seu ambiente. Bebês, desde cedo, apresentam algum tipo de capacidade mnemônica permitindo que possam reconhecer determinados objetos e faces humanas. Crianças com idade pré-escolar, com o advento da verbalização, intensificação das suas relações sociais e a maturação do sistema nervoso, organizam seu pensamento e apresentam memória mais elaborada.

As memórias episódica e semântica, assim como a memória de trabalho, apresentam um melhor funcionamento conforme a criança cresce. Com o passar do tempo, a criança vai adquirindo um desempenho mais próximo ao dos adultos, retendo mais informações e acessando-as com maior facilidade. Sistemas de memória implícitos, como a memória de procedimento, presente desde cedo, não apresentam muitas mudanças ao longo do desenvolvimento.

A partir de um conhecimento acerca do desenvolvimento desses diferentes sistemas mnemônicos, é possível planejar trabalhos focais para que haja prevenção de problemas futuros, além de melhor treinamento e estimulação no âmbito educacional e orientações de qualidade às famílias. Essa fase da vida, época em que a criança apresenta grande capacidade de mudanças, representa o momento mais adequado para que processos de estimulação possam ser implementados, permitindo, assim, medidas profiláticas que favoreçam um desenvolvimento mais saudável e mais adaptativo de crianças que eventualmente possam apresentar dificuldades mnemônicas e consequentes problemas de aprendizagem.

\section{Referências bibliográficas}

Abbema, D. L. V., \& Bauer, P. J. (2005). Autobiographical memory in middle childhood: Recollections of the recent and distant past. Memory, 13(8), 829-845.

Alloway, T. P., Gathercole, S. E., \& Pickering, S. J. (2006). Verbal and Visuospatial Short-Term and Working Memory in Children: Are They Separable? Child Development, 77(6), 1.698-1.716.

Ashcraft, M. H. \& Kirk, E. P. (2001). The relationships among working memory, math anxiety, and performance. Journal of Experimental Psychology: General, 130, 224-37.

Baddeley, A. (2000). The episodic buffer: a new component of working memory? Trends in Cognitive Sciences, 4(11), 417-423.

Baddeley, A. (2002). Is Working Memory Still Working? European Psychologist, 7(2), 85-97.

Baddeley, A. (2003). Working Memory: looking back and looking forward. Nature Reviews Neuroscience, 4(10), 829-839.

Baddeley, A. (2007). Working Memory, Thought, and Action. Oxford Psychology Series, Oxford University Press. 
Baddeley, A. (2009). Working Memory. Em A. Baddeley, M. W. Eysenck, \& M. C. Anderson (Eds.), Memory (pp. 41-68). New York: Psychology Press.

Barnat, S. B., Klein, P. J., \& Meltzoff, A. N. (1996). Deferred Imitation Across Changes in Context and Object: Memory and Generalization in 14-Month-old Infants. Infant Behavior and Development, 19(2), 241-251.

Barr, R., Dowden, A., \& Hayne, H. (1996). Developmental Changes in Deferred Imitation By 6- to 24-Month-Old Infants. Infant Behavior and Development, 19(2), 159-170.

Bauer, P. J. (2006). Constructing a past in infancy: a neuro-developmental account. Trends in Cognitive Sciences, 10(4), 175-181.

Bauer, P. J. (2008). Toward a Neuro-Developmental Account of the Development of Declarative Memory. Developmental Psychobiology, 50(1), 19-31.

Bauer, P. J., \& Souci, P. S. (2010). Going beyond the facts: Young children extend knowledge by integrating episodes. Journal of Experimental Child Psychology, 107(4), 452-465.

Bauer, P. J., Kroupina, M. G., Schwade, J. A., Dropik, P. L., \& Wewerka, S. S. (1998). If memory serves, will language? Later verbal accessibility of early memories. Development and Psychopathology, 10(4), 655679

Bauer, P. J., Wenner, J. A., \& Kroupina, M. G. (2002). Making the Past Present: Later Verbal Accessibility of Early Memories. Journal of Cognition and Development, 3(1), 21-47.

Blair, C., \& Diamond, A. (2008). Biological processes in prevention and intervention: the promotion of self-regulation as a means of preventing school failure. Development and Psychopathology, 20(3), 899-911.

Blunden, S., Lushington, K., Kennedy, D., Martin, J., \& Dawson, D. (2000). Behavior and Neurocognitive Performance in Children Aged 5-10 Years Who Snore Compared to Controls. Journal of Clinical and Experimental Neuropsychology, 22(5), 554-568.

Brehmer, Y., Li, S., Müller, V., Von Oertzen, T., \& Lindenberger, U. (2007). Memory Plasticity Across the Life Span: Uncovering Children's Latent Potential. Developmental Psychology, 43(2), 465-478.

Burch, M. M., Austin, J., \& Bauer, P. J. (2004). Understanding the emotional past: Relations between parent and child contributions in emotionally negative and nonnegative events. Journal of Experimental Child Psychology, 89(4), 276-297.

Carneiro, M. P. (2007). Desenvolvimento da Memória na Criança: o que muda com a Idade? Psicologia: Reflexão e Crítica, 21(1), 51-59.

Carver, L. J., Bauer, P. J., \& Nelson, C. A. (2000). Associations between infant brain activity and recall memory. Developmental Science, 3(2), 234-246.

Conway, M. A. (2009). Episodic memories. Neuropsychologia, 47(11), 2.305-2.313

Conway, M. A., \& Pleydell-Pearce, C. W. (2000). The Construction of Autobiographical Memories in the Self-Memory System. Psychological Review, 107(2), 261-288.

Courage, M. L., \& Howe, M. L. (2002). From Infant to Child: The Dynamics of Cognitive Change in the Second Year of Life. Psychological Bulletin, 128(2), 250-277.

De Smedt, B., Janssen, R., Bouwens, K., Verschaffe,1 L., Boets, B., \& Ghesquière, P. (2009). Working memory and individual differences in mathematics achievement: a longitudinal study from first grade to second grade. Journal of Experimental Child Psychology, 103(2), 186-201.

DiGiulio, D. V., Seinderberg, M., O’Leary, D. S., \& Raz, N. (1994). Procedural and Declarative Memory: A Developmental Study. Brain and Cognition, 25(1), 79-91.

Duncan, E. M., Whitney, P., \& Kunen, S. (1982). Integration of Visual and Verbal Information. Child Development, 53(5), 1.215-1.223.

Fivush, R. (2011). The Development of Autobiographical Memory. Annual Review of Psychology, 62, 559-582.

Fivush, R., \& Nelson, K. (2004). Culture and Language in the Emergence of Autobiographical Memory. Psychological Science, 15(9), 573-577.

Fivush, R., \& Schwarzmueller, A. (1998). Children Remember Childhood: Implications for Childhood Amnesia. Applied Cognitive Psychology, 12(5), 455-473.

Gathercole. S. E., Hitch, G. J., Service, E., \& Martin, A. J. (1997). Phonological Short-Term Memory and New Word Learning in Children. Developmental Psychology, 33(6), 966-979.

Gathercole, S. E., Pickering, S. J., Ambridge, B., \& Wearing, H. (2004). The Structure of Working Memory From 4 to 15 Years of Age. Developmental Psychology, 40(2), 177-190.

Gazzaniga, M. S., \& Heatherton, T. F. (2005). Memória (M. A. V. Veronese, Trad.). Em M. S. Gazzaniga, \& T. F. Heatherton, Ciência psicológica: mente, cérebro e comportamento (pp. 214-247). Porto Alegre: Artmed.

Greenbaum, J. L., \& Graf, P. (1989). Preschool period development of implicit and explicit remembering. Bulletin of the Psychonomic Society, 27(5), 417-420.

Greenberg, D. L., \& Verfaellie, M. (2010). Interdependence of episodic and semantic memory: evidence from neuropsychology. Journal of the International Neuropsychological Society, 16(5), 748-753.

Greve, A., van Rossum, M. C., \& Donaldson, D. I. (2007). Investigating the functional interaction between semantic and episodic memory: convergent behavioral and electrophysiological evidence for the role of familiarity. Neuroimage, 34(2), 801-814.

Han, J. J., Leichtman, M. D., \& Wang, Q. (1998). Autobiographical Memory in Korean, Chinese, and American Children. Developmental Psychology, 34(4), 701-713.

Hartshorn, K., \& Rovee-Collier, C. (2003). Does Infant Memory Expression Reflect Age at Encoding or Age at Retrieval? Developmental Psychobiology, 42(3), 283-291.

Kandel, E. R. (2009). The biology of memory: a forty-year perspective. The Journal of Neuroscience, 29(41), 12748-12756.

Korkman, M., Kemp, S. L., \& Kirk, U. (2001). Effects of Age on Neurocognitive Measures of Children Ages 5 to 12: A CrossSectional Study on 800 Children from the United States. Developmental Neuropsychology, 20(1), 331-354.

Kramer, J. H.; Delis, D. C.; Kaplan, E.; O'Donnell, L.; \& Prifitera, A. (1997). Developmental sex differences in verbal learning. Neuropsychology, 11(4), 577-584

Landeira-Fernandez, J. (2006). Amnésias. Em Brandão, M. L. \& Graeff, F. G. (Orgs.), Neurobiology of Mental Disorders (pp. 157-187). Nova York: Nova Publishers.

Larkina, M., Güler, O. E., Kleinknecht, E., \& Bauer, P.J. (2008). Maternal provision of structure in a deliberate memory task in relation to their preschool children's recall. Journal of Experimental Child Psychology, 100(4), 235-251.

Lombroso, P. (2004). Aprendizado e memória. Revista Brasileira de Psiquiatria, 26(3), 207-210.

Lukowski, A. F.,Wiebe, S. A., Haight, J. C., DeBoer, T., Nelson, C. A., \& Bauer, P. J. (2005). Forming a stable memory representation in the first year of life: why imitation is more than child's play. Developmental Science, 8(3), 279-298.

Luria, A. H. (1981). Memória. Em A. H. Luria, Fundamentos de Neuropsicologia (pp. 245-265). São Paulo: Edusp.

Maehler, C., \& Schuchardt, K. (2009). Working memory functioning in children with learning disabilities: does intelligence make a difference? Journal of Intellectual Disability Research, 53(1), 3-10.

McKee, R. D., \& Squire, L. R. (1993). On the development of declarative memory. Journal of Experimental Psychology: Learning, Memory, and Cognition, 19(2), 397-404.

Mello, C. B. \& Xavier, G. F. (2006). Desenvolvimento da memória: influências do conhecimento de base e do uso de estratégias. Em C. B. Mello, M. C. Miranda, \& M. Muszkat, Neuropsicologia do desenvolvimento: conceitos e abordagens (pp. 93-105). São Paulo: Memnon.

Meltzoff, A. N. (1988). Infant Imitation and Memory: Nine-Month-Olds in Immediate and Deferred Tests. Child Development, 59(1), 217-225.

Morrison, C. M., \& Conway, M. A. (2010). First words and first memories. Cognition, 116(1), 23-32.

Moscovitch, M., Rosenbaum, R. S., Gilboa, A., Addis, D. R., Westmacott, R. Grady, C. McAndrews, M. P. Levine, B. Black, S. Winocur, G. \& Nadel, L. (2005). Functional neuroanatomy of remote episodic, semantic and spatial memory: a unified account based on multiple trace theory. Journal of Anatomy, 207(1), 35-66.

Nelson, C. A. (1995). The Ontogeny of Human Memory: A Cognitive Neuroscience Perspective. Developmental Psychology, 31(5), 723738 .

Nelson, C. A. (1998). The Nature of Early Memory. Preventive Medicine, 27(2), 172-179

Nelson, K. (2007). Development of extended memory. Journal of Physiology - Paris, 101(4-6), 223-229.

Nelson, C. A., \& Collins, P. F. (1991). Event-Related Potential and LookingTime Analysis of Infants' Responses to Familiar and Novel Events: Implications for Visual Recognition Memory. Developmental Psychology, 27(1), 50-58.

Nelson, K., \& Fivush, R. (2004). The Emergence of Autobiographical Memory: A Social Cultural Developmental Theory. Psychological Review, 111(2), 486-511. 


\section{DESENVOLVIMENTO DA MEMÓRIA}

Reese, E., Haden, C. A., \& Fivush, R. (1996). Mothers, fathers, daughters, sons: gender differences in autobiographical reminiscing. Research on Language and Social Interaction, 29(1), 27-56.

Rovee-Collier, C., Hartshorn, K., \& DiRubbo, M. (1999). Long-Term Maintenance of Infant Memory. Developmental Psychobiology, 35(2), 91-102.

Rovee-Collier, C., \& Cuevas, K. (2009). Multiple memory systems are unnecessary to account for infant memory development: an ecological model. Developmental Psychology, 45(1), 160-174.

Squire, L. R., \& Zola-Morgan, S. (1991). The Medial Temporal Lobe Memory System. Science, 253(5026), 1.380-1.386.

Squire, L. R., Ojemann, J. G., Miezin, F. M., Petersen, S. E., Videen, T. O., \& Raichle, M. E. (1992). Activation of the Hippocampus in Normal Humans: a functional anatomical study of memory (amygdala/declarative memory/priming/frontal cortex). Neurobiology, 89(5), 1.837-1.841.

Towse, J. N., Hitch, G. J., \& Hutton, U. (2002). On the nature of the relationship between processing activity and item retention in children. Journal of Experimental Child Psychology, 82(2), 156-184.

Tulving, E. (2002). Episodic Memory: From Mind to Brain. Annual Review of Psychology, 53, 1-25.

Uehara, E. \& Landeira-Fernandez, J. (2010). Um panorama sobre o desenvolvimento da memória de trabalho e seus prejuízos no aprendizado escolar. Ciências e Cognição, 15(2), 031-041.

Uema, S. F. H., Pignatari, S. S. N., Fujita, R. R., Moreira, G. A., PradellaHallinan, M., \& Weckx, L. (2007). Avaliação da função cognitiva da aprendizagem em crianças com distúrbios obstrutivos do sono. Revista Brasileira de Otorrinolaringologia, 73(3), 315-320.

Usher, J. A. \& Neisser, U. (1994). Childhood amnesia and the beginnings of memory for four early life events. Journal of Experimental Psychology: General, 122(2), 155-165.

Wilburn, C., \& Feeney, A. (2008). Do development and learning really decrease memory? On similarity and category-based induction in adults and children. Cognition, 106(3), 1.451-1.464. 